\title{
Control Strategy of Micro Inverter based on Improved MPPT
}

\author{
Chunchao $\mathrm{Yu}^{1,{ }^{*}}$, Debiao $\mathrm{Du}^{2}$, Xiaoxu $\mathrm{Yu}^{3}$, Haisen $\mathrm{Ji}^{3}$, Min Yan ${ }^{1}$, \\ and Zhenggang Lei ${ }^{1}$ \\ ${ }^{1}$ kunming institute of physics, Kunming 650223 \\ ${ }^{2}$ Yunnan Provincial Hospital of traditional Chinese Medicine, Kunming 650021 \\ ${ }^{3}$ State Grid Liaoyang Electric Power Supply Company Information\& Telecommunication Branch, \\ Liaoning 111000, China
}

Keywords: Photovoltaic power generation; Improved MPPT algorithm; Micro inverter; interleaved flyback.

\begin{abstract}
The paper focuses on the photovoltaic inverter MPPT algorithm. The traditional maximum power point tracking algorithm development has been simole told. Based on the traditional perturbation and observation method and combined linear fitting method, the MPPT algorithm has been improved. The simulation experiment in MATLAB shows that the algorithm is feasible.
\end{abstract}

\section{Introduction}

In the development and application of micro inverter, most of them are in the photovoltaic power generation system. The research of solar photovoltaic power generation system is actually the photoelectric conversion efficiency. So the algorithm of maximum power point tracking is researched. Because the research on the control strategy can also greatly improve the inverter in photovoltaic power generation. In the study of MPPT algorithm, more factors are considered, such as illumination intensity, illumination time, environment temperature and other factors that affect the PV panel side. It is precisely because of the influence of different factors, solar photovoltaic power generation has a maximum power output, which is called the MPPT point. In order to maximize the maximum power output and light energy conversion, the MPPT algorithm is involved.

MPPT algorithm is the traditional maximum power point tracking, more extensive use of the disturbance observer method and incremental conductance method, the main control is by controlling the output voltage and current of PV components, real-time closed-loop sampling control. Research shows that for the MPPT algorithm in the two self optimizing class, is usually used for voltage or current step or variable step search. Send a is calculated and comparison process, real-time detection of the current state of voltage and current, the output power of the sample, and then power have stored with the previous comparison, comparison of Deshaus stay small, this is a real time comparison, has been compared. To try to approximate the output circuit of photovoltaic maximum power point, this is the traditional self optimization algorithm flow 2 micro inverter MPPT control strategy.

\section{Improved MPPT Algorithm Control Strategy}

Usually in the photovoltaic power generation system, there are many factors that affect the efficiency of photovoltaic power generation, such as the hardware and control strategy of the internal characteristics of the system, the main circuit and is influenced by the environmental factors (temperature, partial shadow area, light intensity etc.).

This paper presents an improved MPPT algorithm based on the perturbation and observation, this algorithm can overcome the shortcomings of traditional perturbation and observation method, fast and accurately track the maximum power point, improve the efficiency of power generation, the simulation experiments verify the effectiveness of the algorithm. 


\subsection{Linear Fitting and Dual-Mode Control Algorithm Pressure Disturbance Observation}

The traditional perturbation and observation method, MPPT algorithm is more popular in recent years, through the comparison of the maximum power point variable step does not stop, this is the biggest problem in the vicinity of the maximum power point will appear a disturbance, the disturbance of climbing such long time will lead to the photoelectric conversion efficiency is relatively low. Even though fluctuations can be reduced by decreasing the perturbation step length, the sensitivity of the system is not sufficient to cope with changes in climate temperature with the extension of the tracking time. Therefore, the step size selection for perturbation observation should be a compromise between faster tracking and less fluctuation.

In the circuit model, the solar cell $R_{s h}$ with a single section high shunt resistance $\left(n_{s}=1, n_{p}=1\right)$, the power of the photovoltaic array can be estimated according to the formula (1).

$$
p_{p v}=\alpha I_{p v} \cdot \operatorname{In}\left[\frac{\left(I_{p h}+I_{s a t}-I_{p V}\right)}{I_{s a t}}\right]-I_{P V}^{2} R_{s}
$$

Among them, $\alpha=(k . T . A) / q$, when the MPP point of $\alpha$ is zero, then calculate the partial derivative of $I_{p v}$, you can launch the corresponding constraint MPP points of the equation formula (2):

$$
p_{p v}-\alpha I_{p v} \cdot I n\left[\frac{\alpha I_{P V}^{2}}{\left(p_{p v}-I_{P V}^{2} R_{s}\right) I_{s a t}}\right]+I_{P V}^{2} R_{s}=0
$$

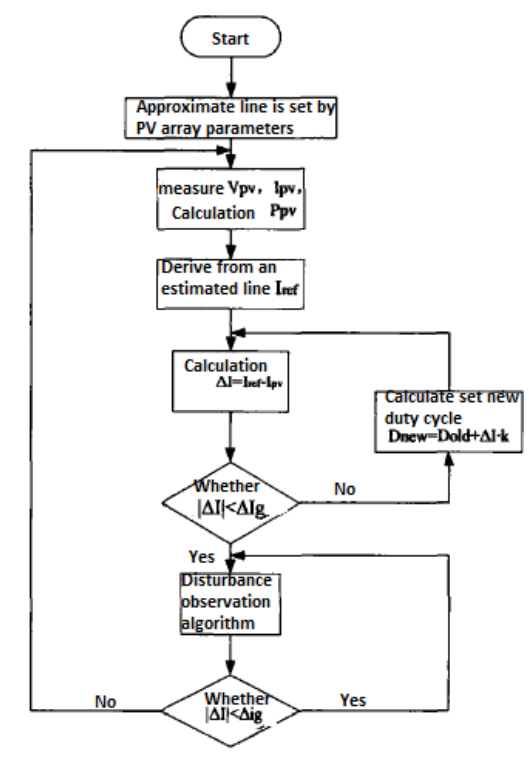

Fig. 1 control flow chart of the new algorithm

Fig. 1 gives the control flow chart of the new algorithm. In combination with the two MPPT algorithms, the first step is to estimate the approximate line of the maximum power point through the given electrical parameters. The reference quantity of the current $I_{r e f}$ is determined by the voltage and current of the PV plate obtained by the linear combination measurement. The duty cycle of the PWM waveform of the drive switching device is generated by the difference of current $I_{p v}$ and $I_{\text {ref }}$ produced by the PV board $\Delta I$. When the current difference $\Delta I$ is small enough, this means that the point of operation is already close to the theoretical MPPT point. It needs to be converted into the control step disturbance observation method, in order to send can continue to track the maximum power point in the condition of dynamic, accurately find out the maximum power point of need, if there is short-term weather fluctuations caused by the sudden expansion of $\Delta I$, the proposed control algorithm to control the linear fitting step back again. To $\Delta I$ again become small enough so far. 


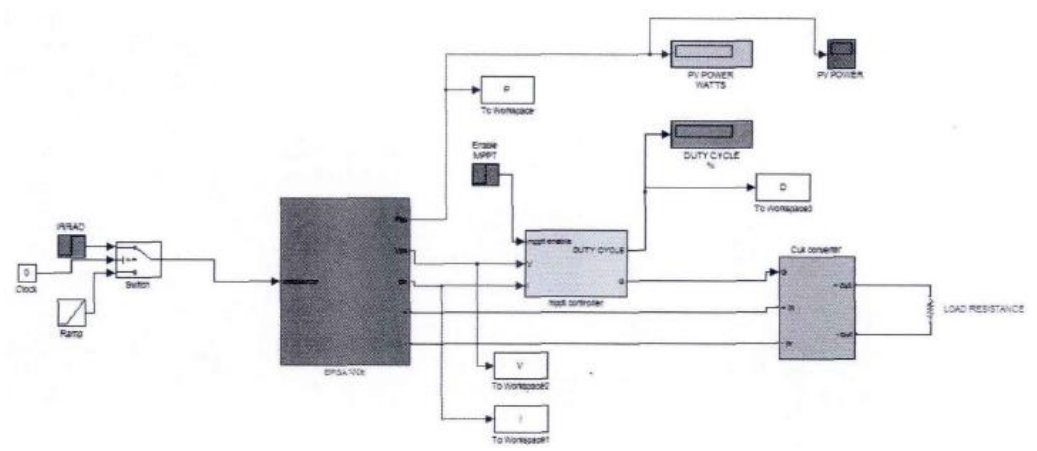

Fig.2 Simulation model diagram

The simulation experiment figure 2 is conducted using simulation platform given above, at the temperature of $25 \mathrm{oC}$ and the change of light intensity, the characteristic curves obtained with this simulation is the same, then the maximum power point under different intensities of light by linear fitting out. The maximum power point of the simulation is connected in a straight line, and the relationship can be seen intuitively from the following Fig. 3 results.

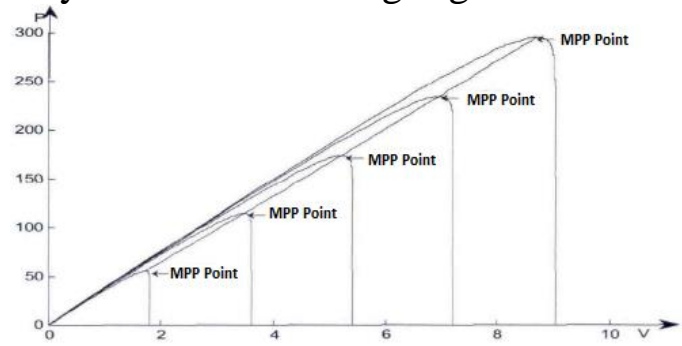

Fig.3 Under different lights $25 \mathrm{oC}$ of new algorithm simulation diagram

By building the Simulink simulation platform, the characteristic curves of PV array under different illumination and temperature are obtained by using the simulation results of the previous two sets. The biggest characteristic of linear fitting is through mathematical calculate angle curve, this simulation experiment can approximate straight line tilt angle of photovoltaic array through the micro network gives the electrical parameters calculation, and the design and the derivation of MPPT algorithm of the digital control circuit is also very easy to achieve. Compared with the traditional process of perturbation and observation method of MPPT, Therefore, compared with the traditional methods, the proposed linear estimation method combined with the traditional perturbation observation has the advantages of high efficiency, less volatility and more accurate. Usually know the most needed in the MPPT algorithm is the time to lock the maximum power point, then the output voltage is needed, the improved algorithm is the use of the superiority of the algorithm, save time, improve the photoelectric conversion efficiency and the effect, will be behind the related simulation verification.

\section{The Pressure Analysis of Simulation Results}

Photovoltaic components related electrical parameters are as follows: maximum power $P=280 \mathrm{~W}$, open circuit voltage $V o c=37.98 \mathrm{~V}$, maximum power point, operating voltage $V m p p=30.63 \mathrm{~V}$, short-circuit current $I m p p=8.49 \mathrm{~A}$, maximum power point, operating current $I s c=9.04 \mathrm{~A}$. Through the experimental data given, the related three group simulation experiments are carried out.

The following three sets of experimental results were analyzed. Fig. 4 shows that the output power $\mathrm{P}$, voltage $\mathrm{U}$ and current $\mathrm{I}$ are obtained under the control of the traditional perturbation observation method at the illumination intensity of $1000 \mathrm{w} / \mathrm{m}^{2}$ and the outdoor temperature of $25 \mathrm{oC}$. From the simulation results, it can be seen that the accuracy is not much different from the latter, but the time spent in tracking the maximum power point is relatively long, and the result is that the inverter efficiency is not high, and the energy consumption is relatively large.

Under the same illumination and temperature conditions, the improved MPPT algorithm is used to simulate the experiment, and the experimental results such as figure 4 are obtained. The improved 
MPPT algorithm can effectively shorten the maximum power point capture time, save energy consumption, improve efficiency, and maintain a high degree of accuracy.
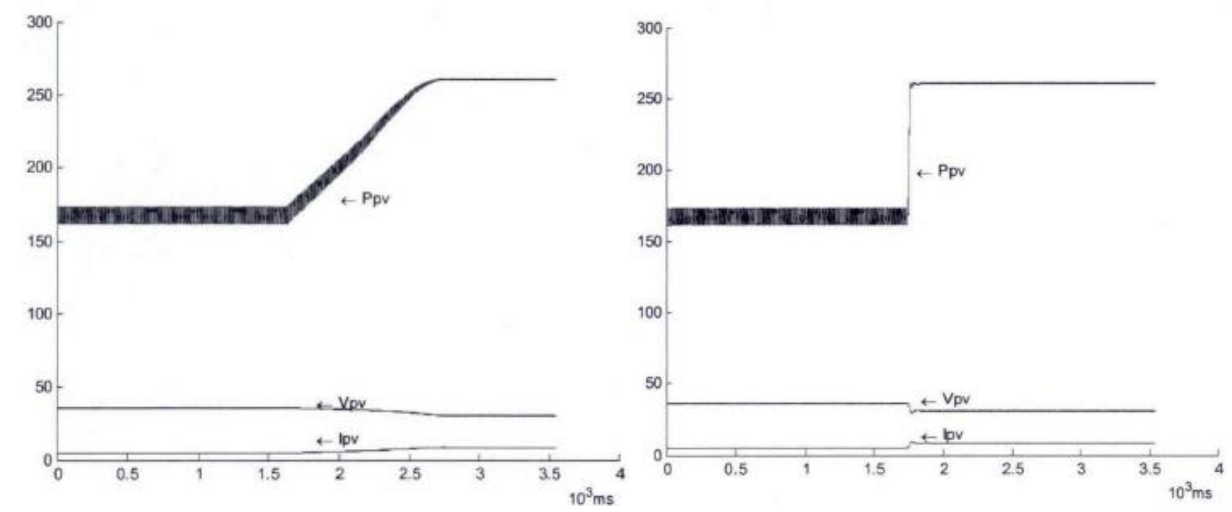

Fig.4 (a)The traditional perturbation observation method and New control algorithm is obtained power P voltage $\mathrm{U}$ and current I

In order to confirm the applicability of the improved MPPT algorithm under different illumination conditions, we do a set of experiments when the illumination intensity is $800 \mathrm{w} / \mathrm{m}^{2}$, the temperature is $30 \mathrm{oC}$, and the other conditions remain unchanged. The simulation results are shown in figure 5 . The improved MPPT algorithm can still find the maximum power point accurately and quickly.

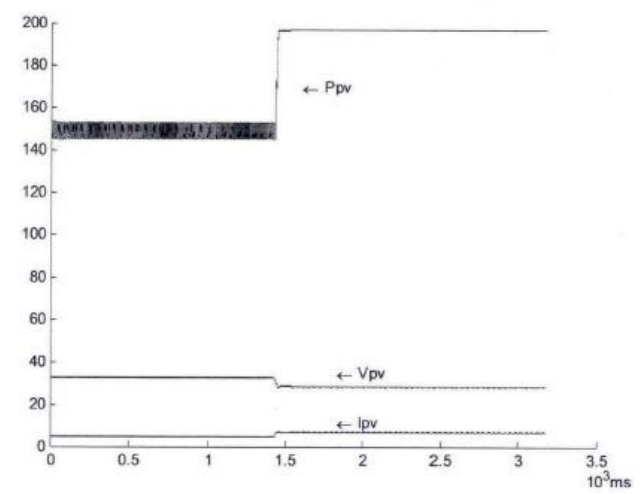

Fig.5 Power P voltage $U$ and current I is obtained under new temperature and illumination conditions

The simulation results show the applicability and effectiveness of the improved algorithm, and the experimental results are expected. The improved algorithm can track the maximum power point quickly and reduce the loss of energy in order to achieve the maximum power generation efficiency.

\section{Conclusion}

The experimental results, it is clear that MPPT algorithm has strong tracking characteristics, can improve the conversion efficiency of the micro inverter, the simulation results are improved in the algorithm can reduce the fluctuation, fast track the maximum power point, thereby reducing energy losses, to achieve the goal of maximum power generation efficiency.

\section{References}

[1]. Zongxiang Lu, Wang Caixia, Kuo Yong. Research on Microgrid overview of [J] power electronic automation, 2007, 31 (19); 100-107.

[2]. Wang Chengshan, Xiao Zhaoxia, Wang Shouxiang. Integrated control and analysis of microgrid. [J]. automation of electric power systems, 2008, 32 (7): 98-103.

[3]. Zhou Lin, Wu Jian, Li Qiuhua, et al. Overview of PV array maximum power point tracking control method [J] high voltage technology, 2008,34 (6): 1145-1154.

[4]. Yang Haizhu, Jin Xinmin. An improved measure of maximum power point tracking control of grid connected photovoltaic system and its simulation and experimental study [J] new technology of electrical and electrical energy, 2006,25 (1) 63-67. 
[5]. Li as Zhou Lin, Liu Qiang. The new algorithm and Simulation of [J]. Electric power automation equipment maximum power tracking photovoltaic power systems, 2008,28 (7): 21-25.

[6]. . Jiao Yang, song Qiang, Liu Wenhua. Control strategy of photovoltaic grid connected system based on improved algorithm [J]. Electric power automation equipment, 2010,30 (12): 92-96. 